\title{
DIFFERENTIAL DIAGNOSIS OF FACIAL AND DENTAL MANIFESTATIONS IN CONGENITAL SYPHILIS
}

\author{
BY \\ G. O. HORNE \\ From the Department of Venereal Diseases, General Infirmary at Leeds
}

(RECEIVED FOR PUBLICATION NOVEMBer 4, 1953)

A confident diagnosis of congenital syphilis can sometimes be made in children who appear otherwise normal from inspection of the facies and the mouth. Indeed, the observant doctor or dentist has in this way frequently been the means of discovering syphilis in a family. On the other hand, it is possible to mistake one or other of several conditions which in their facial and dental manifestations simulate congenital syphilis, and which, in view of the diminishing incidence of this disease, are likely to become relatively more common. This differential diagnosis is rarely referred to in textbooks on syphilology or paediatrics.

It is important that if there is syphilis in a family it should be discovered as early as possible so that those infected may have the benefit of treatment, and to prevent further members of the family becoming involved. But, for obvious reasons, it is equally important that congenital syphilis should not be wrongly diagnosed, and that it should be confidently rejected if it is not present. A high index of suspicion should be countered with great care so as not to make a diagnosis of syphilis negligently.

There are several conditions that mimic congenital syphilis in their manifestations in the face and mouth. The most confusing of these is the anhidrotic form of hereditary ectodermal dysplasia, which, the literature reveals, has frequently been misdiagnosed as congenital syphilis. In this condition the facies can be very misleading. The bridge of the nose is depressed so that it has a saddle-shaped appearance; the brow is high and wide; there may be scars on the face similar to the classical rhagades of congenital syphilis; and there are usually dental abnormalities, particularly of the permanent incisors and canines.

Many of the photographs of the faces of such cases in the published papers on the subject are almost indistinguishable from those seen in congenital syphilis, and the matter is further complicated by the fact that several members of a family may be affected. Careful observation, however, reveals several important differences. In hereditary ectodermal dysplasia there is no bossing of the frontal bones, but the depressed bridge of the nose may be exaggerated after puberty by prominent supraorbital ridges. The 'pseudo-rhagades' radiate round the nostrils rather than the mouth, as is the case in congenital syphilis, and the lips tend to be thickened. The dental abnormalities are usually gross: there may be anodontia, or only a few teeth; there is gross disorder in the time of their eruption; many of the teeth, except the molars, are abnormally shaped, usually being small and conical, but they never show the morphological characteristics sometimes seen in congenital syphilis.

Diagnostic, however, in the fully developed syndrome is the absence of sweat glands-the most important component-the subjects thus being intolerant of heat. Also, in most cases, the head hair tends to be sparse and fine, and the eyebrows sparse; body hair may be deficient or absent. The nails may also show minor or gross defects.

Dental dysplasias, with little or no evidence of ectodermal involvement, may also cause confusion. However, careful examination of abnormally shaped teeth will reveal the differences between congenital syphilis and other dental dysplasias in which the typical Hutchinsonian tooth and its variants and Moon's (6-year-old) molars do not occur. Disordered timing of eruption and hypodontia are not features of congenital syphilis.

Reviews of the various types of hereditary ectodermal dysplasias include those by MacKee and Andrews (1924), Smith (1929), Cockayne (1933), and de Silva (1939). The dental abnormalities of congenital syphilis are well described in the illustrated paper by Cruickshank (1939).

The depressed bridge of the nose is a notorious stigma of congenital syphilis, but there are other conditions in which it occurs as well as the one already discussed. It is one of the features of gargoylism, but in this condition other signs, such 
as mental defect and osteochondrodystrophy, usually make the diagnosis unequivocal; however, the enlarged liver and spleen and the cloudy cornea of gargoylism may raise doubts, although the nature of the corneal lesion is quite different from the interstitial keratitis of congenital syphilis. In the formes frustes of gargoylism some of the gross features may be absent, and differential diagnosis rendered more difficult if the bridge of the nose is depressed.

In achondroplasia the base of the skull is underdeveloped, and the normal development of the vertex causes the forehead to be relatively prominent, and so even exaggerate any depression of the bridge of the nose that may be present. However, other manifestations, particularly the diminished stature, usually make the diagnosis straightforward.

Depression of the bridge of the nose can be present without any other bodily abnormality. There is a considerable range of normal profiles in which the bridge of the nose is depressed, but there is a developmental anomaly which occurs by itself and which may lead to suspicion of congenital syphilis. This is partial or complete absence of the nasal bone (see Case 1).

In major conditions, such as the anhidrotic form of hereditary ectodermal dysplasia and gargoylism, clinical differentiation from congenital syphilis should seldom be difficult. It is in the minor conditions that confusion may occur, especially when other circumstances combine to aggravate the problem. The following two case histories illustrate the type of difficulty. They also illustrate the steps that sometimes require to be taken in order to eliminate congenital syphilis, bearing in mind that in this condition, especially in older children, serological tests are occasionally equivocal or even negative.

\section{Case Histories}

Case 1. A boy, aged 6, while under periodic observation for severe bilateral myopia developed cyclitis in the right eye associated with corneal oedema. Topical cortisone was started immediately and rapidly controlled the condition. The possibility of interstitial keratitis was considered, although the inflammation was arrested before there was time for the characteristic appearances to develop. A possible syphilitic aetiology was supported by the boy's facies. The blood Wassermann reaction was negative, but he was referred for an opinion.

Apart from the progressive myopia there was nothing significant in the previous history; there was no evidence of healed or active choroiditis. He had had recurrent "colds" for several years, but at no time was there any unusual nasal discharge.

He was of normal physical and mental development for his age. The facies is illustrated in Figs. 1 and 2. The

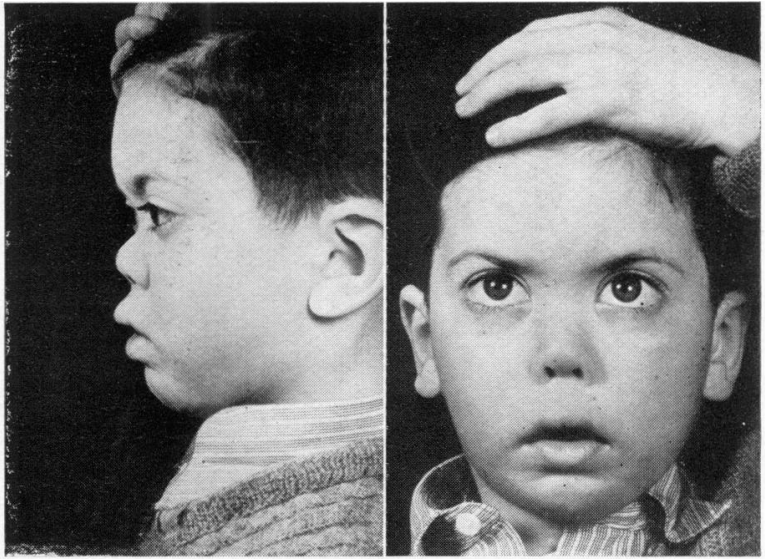

FIGS. 1 and 2.-Facies showing depressed bridge of nose.

most striking feature was the depressed, "saddleshaped" type bridge of the nose; the forehead was prominent, but the supraorbital ridges were normal and there was no frontal bossing. The maxilla appeared to be slightly foreshortened, but there was no abnormality of the palate or dental arch. In spite of the nasal outline the facies did not give the general impression of being typical of congenital syphilis. The temporary dentition and the first permanent molars were all normal. Palpation revealed a deficient cartilaginous septum of the nose (Fig. 3). The nasal mucous membrane was normal, and there was no evidence of recent or past ulceration or destruction of tissues of the nose. No signs of syphilis were found elsewhere, nor was there clinical evidence of any other disease. Sweating function was normal.

The routine blood Wassermann reaction was negative on two further occasions, and the cardiolipin Wassermann reaction, Kahn test, and Price's precipitation reaction were also negative. Radiographs of the wrists, elbows, and spine showed no abnormality. A radiograph of the skull showed that there was no nasal bone; the relatively horizontal angle of the unerupted

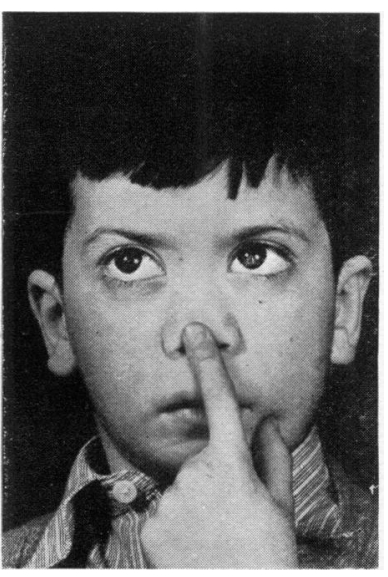

FIG. 3.-Photograph illustrating deficient cartilagenous nasal septum. upper incisors suggested some foreshortening of the maxilla, but no other abnormality was observed (Fig. 4). An intra-oral film showed that the unerupted upper central incisors were normal in shape.

The boy's mother and father both appeared to be healthy, and had normal facies. Neither was aware of any relative having a nasal deformity similar to that of their child. The mother had had a miscarriage before the 


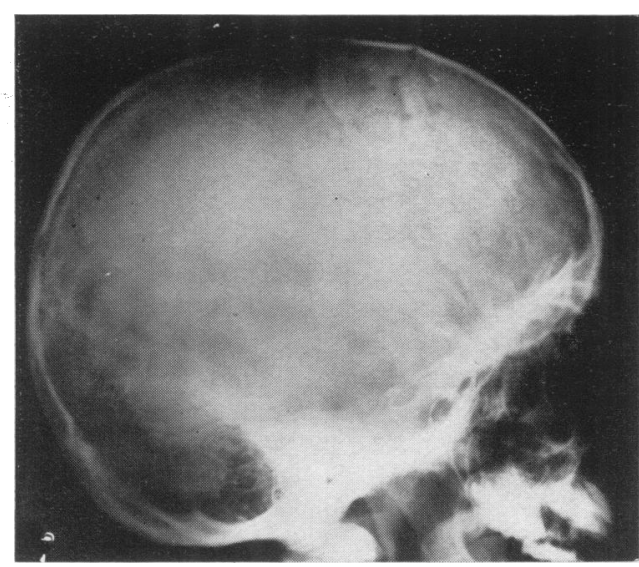

FIG. 4.-Radiograph of skull showing absence of nasal bone.

birth of the living child, and there had been no other pregnancies. The blood Wassermann reactions of the mother and father were negative.

It was decided that the child did not have syphilis. Topical cortisone was continued for several weeks, and there was no relapse of the inflammation on its withdrawal. No other treatment was given. The child has remained under periodic observation for a year, and there have been no further developments.

In this case the nasal deformity was ignored until the development of an inflammatory eye condition, when the combination of the two raised the necessity of considering a diagnosis of congenital syphilis. This condition was confidently ruled out, and the nasal deformity was found to be due to absence of the nasal bone. (Incidentally, this case illustrates the difficulty that may arise from the prompt use of topical cortisone in an inflammatory condition of the eye in children. The latter may never develop far enough to allow a clinical diagnosis, for example, syphilitic interstitial keratitis, to be confidently made. This disadvantage is, of course, outweighed by the beneficial local effect.)

Case 2. A boy, aged 14, was referred with a tentative diagnosis of congenital syphilis which had been made on the grounds of an abnormal dentition, supported by the results of serological tests on the boy and on his mother. He appeared to be healthy, and was well developed for his age (height, $5 \mathrm{ft} .7 \mathrm{in}$., weight, $140 \mathrm{lb}$.). The facies was normal, and there were no obvious stigmata of congenital syphilis. The dentition was grossly abnormal: a number of deciduous teeth were still present, some of the permanent teeth were missing, and some of those present were abnormal in shape (Figs. 5 and 6). The only two permanent upper incisors, however, were not Hutchinsonian, nor did they resemble any of the variants sometimes seen in congenital syphilis. Two of the lower incisors tended to be "screwdriver-shaped" and the lower canines were conical. The permanent molars were all normal, and one temporary molar was still present.

The hair on the scalp was rather sparse, and of fine texture; the eyebrows were sparse, and the pubic hair fine (Fig. 7). The nails and sweating function were normal. Clinical examination revealed no other abnormalities. Radiographs of the wrists and elbows showed no evidence of past or present disease of the bones. A radiograph of the jaws showed that some of the permanent teeth were unerupted and some were absent.

The boy had always been healthy, and there was nothing significant in the previous history, except that the first teeth did not appear until he was 3 years old. His mother appeared healthy, and showed no clinical evidence of disease. Her first husband died at the age of 27 from lobar pneumonia, and she had married again. There had been no children since her second marriage. There was one other child, a girl aged $15 \frac{1}{2}$, who was also healthy. The mother's and daughter's dentitions were normal.

The original report on the serological tests on both the boy and his mother stated that the Wassermann

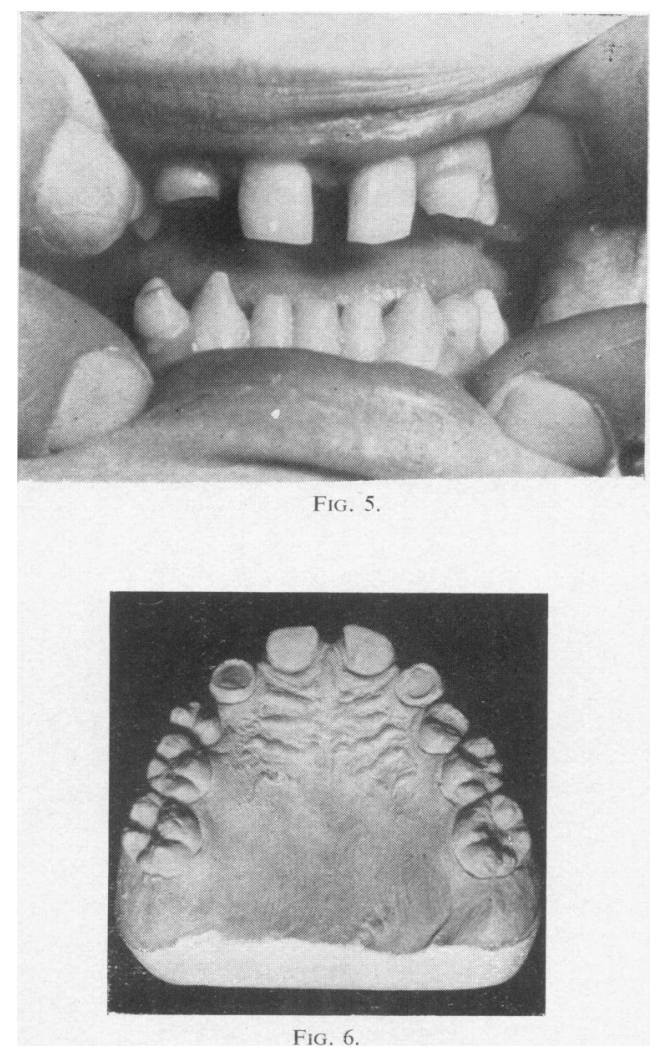

FIGS. 5 and 6.-Dental abnormalities.

The cast of the upper jaw was made at the age of 14 , and the other photograph was taken two years lateı, but there had been no change in the dentition in the interval. 


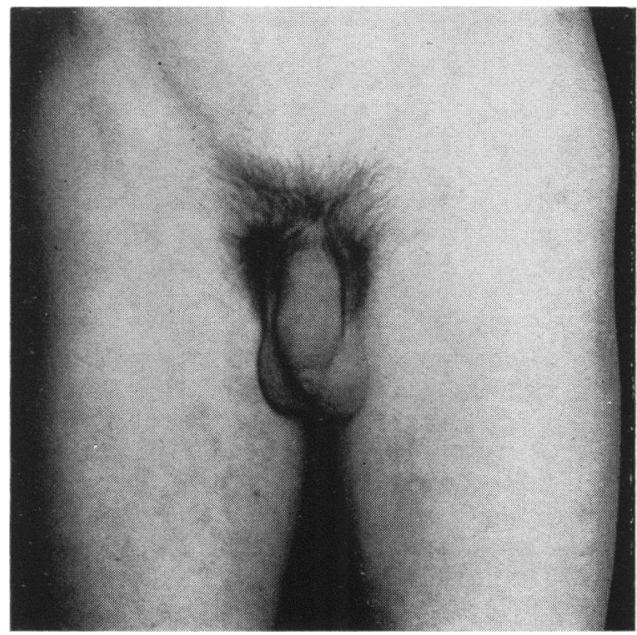

FIG. 7.- Photograph illustrating the sparse, fine pubic hair taken when the boy was 16 years old. There had been very little change in the appearance and distribution of the hair over the previous two years.

reaction was weakly positive and the Kahn test negative. These were repeated at two different laboratories and the Wassermann reaction and Kahn tests were reported negative in all instances. The sister's tests were also negative. The boy's Wassermann reaction and Kahn test were repeated four times during the subsequent six months, and on every occasion they were both reported negative. The report on the cerebrospinal fluid was: cells, 1 per c.mm.; protein, $20 \mathrm{mg}$. \%; Wassermann reaction, negative; Lange curve, normal.

It was decided that the boy did not have syphilis, and no treatment was given. He has been observed at intervals over a period of five years. No further teeth have erupted, and a partial denture has been substituted for the unsightly upper front teeth. He is now practically bald, and the pubic hair is of approximately the same distribution and texture as when he was first seen at the age of 14. Otherwise he has developed normally.

\section{Comment}

In this case inspection of the teeth had prompted serological testing for syphilis. The results of these tests, and of similar tests on the boy's mother, were equivocal, but together were sufficient to arouse suspicion of syphilis. However, the dental abnormalities were not of the type associated with congenital syphilis, and no clinical evidence of this disease could be found. Repeated serological testing ruled out syphilis. The only other evidence of ectodermal defect was the slightly abnormal head and body hair. (Incidentally, this case illustrates the desirability of repeating serological tests for syphilis before drawing conclusions from them. The original results on both the boy and his mother were presumably errors attributable to some technical reason.)

\section{Summary}

Attention has been drawn to the significance of facial and dental abnormalities in the diagnosis of congenital syphilis. Confusion with syphilis may occur in such conditions as the anhidrotic form of hereditary ectodermal dysplasia; dental dysplasia, with or without other evidence of ectodermal defect; formes frustes of gargoylism and achondroplasia; and congenital maldevelopment or absence of the nasal bone. In view of the diminishing incidence of syphilis, these other diseases will become relatively more common.

Two illustrative case histories are reported in which congenital syphilis was suspected, but eventually ruled out. In one there was congenital absence of the nasal bone, and in the other, dental dysplasia. The cases also illustrate other aspects of the confusion that may occur under these circumstances, and the steps that have to be taken to eliminate congenital syphilis.

I am indebted to Mr. John Foster, F.R.C.S., for referring Case 1.

\section{REFERENCES}

Cockayne, E. A. (1933). Inherited Abnormalities of the Skin and its Appendages. London.

Cruickshank, L. G. (1939). Proc. roy. Soc. Med., 32, 343.

MacKee, G. M. and Andrews, G. C. (1924). Arch. Derm. Syph., Chicago, 10, 673.

Silva, P. C. C. de (1939). Quart. J. Med., 8, 97.

Smith, J. (1929). Archives of Disease in Childhood, 4, 215. 